\title{
In vitro image characteristics of an abdominal aortic stent graft: CTA versus 3D MRA
}

\author{
Paul R. Hilfiker, Harald H. Quick, Michaela Schmidt, Joerg F. Debatin* \\ Institute of Diagnostic Radiology, Unitersity. Hospital Zurich, Ramistr. 100, CH-809I Zurich, Switzerland
}

Received 13 July 1998; received in revised form 11 September 1998; accepted 17 November 1998

\begin{abstract}
Percutaneous stent-grafting is increasingly employed as a less invasive alternative to surgery for the treatment of infrarenal abdominal aortic aneurysms. It requires long-term imaging follow-up, to document the structural integrity of the device, to exclude perigraft channels and endograft leakages, as well as the shrinkage of the aneurysmal sac. The expectation of severe stent induced artifacts and safety concerns have prevented 3D MRA from being used. The purpose of this in vitro study was to investigate the imaging characteristics of a bifurcated stent graft with 3D MRA (3D Fourier transform fast spoiled GRE) at 1.5 $\mathrm{T}$ in comparison to those of CTA. Measurement of the stent wall thickness and luminal diameter were made on a agar gel embedded stent graft at five locations on both CTA and MRA images. The stent graft was depicted as a dark ring on MR images. Wall thickness measurements at the five locations of the stent graft overestimated the true stent thickness, while luminal diameters were slightly underestimated. Measurement differences between MR and CT were not statistically significant $(P=0.67 ; P=0.85)$. Artifacts emanating from the platinum markers were considerably less severe on the MR-images. A wider area of signal loss was seen only at the insertion of the iliac stent leg into the aortic stent portion due to the overlap of two radio-opaque platinum markers. 3D MRA images should permit a comprehensive assessment of the arterial lumen, and of perivascular tissues. (C) 1999 Elsevier Science B.V. All rights reserved.
\end{abstract}

Kevwords: Magnetic resonance (MR); Artifact; Vascular studies; Grafts; Interventional procedure; Stents; Prosthesis

\section{Introduction}

Percutaneous stent-grafting is increasingly employed as a less invasive alternative for the treatment of infrarenal abdominal aortic aneurysms [1-3]. In contrast to surgery, aneurysmal stenting requires long-term imaging follow-up, to document the structural integrity of the device as well as the shrinkage of the aneurysmal sac [4]. The expectation of severe stent induced artifacts and safety concerns have pre-

\footnotetext{
* Corresponding author. Tel.: + 11-1-2555193; fax: + 11-12554443.

E-mail address: debatin@dmr.usz.ch (J.F. Debatin)
}

vented 3D MRA from being used for this follow-up imaging [5].

The purpose of this in vitro study was to compare the imaging characteristics of an aortic stent graft on 3D MRA images to those seen on CT images.

\section{Material and methods}

\subsection{Stent graft}

A commercially available stent graft, used routinely in our institution (Vanguard; Boston Scientific, Oakland, NJ) was studied. The self-expanding endoprosthesis is composed of a nitinol frame annealed into a tubular zigzag configuration by a 7-0 polypropylene 
thread [6] and covered with a $0.1 \mathrm{~mm}$ woven-polyester fabric. The bifurcated device has two components: the larger component consists of an aortic graft with a short, $10 \mathrm{~mm}$ wide iliac branch, into which the smaller component is inserted [7]. Both ends of the stent frame, as well as the junction between the two sections are tagged with a platinum marker allowing proper device positioning under fluoroscopic guidance. The stent used in these experiments was $153 \mathrm{~mm}$ long with an aortic diameter of $24 \mathrm{~mm}$ and an iliac diameter of $10 \mathrm{~mm}$ (Fig. 1).

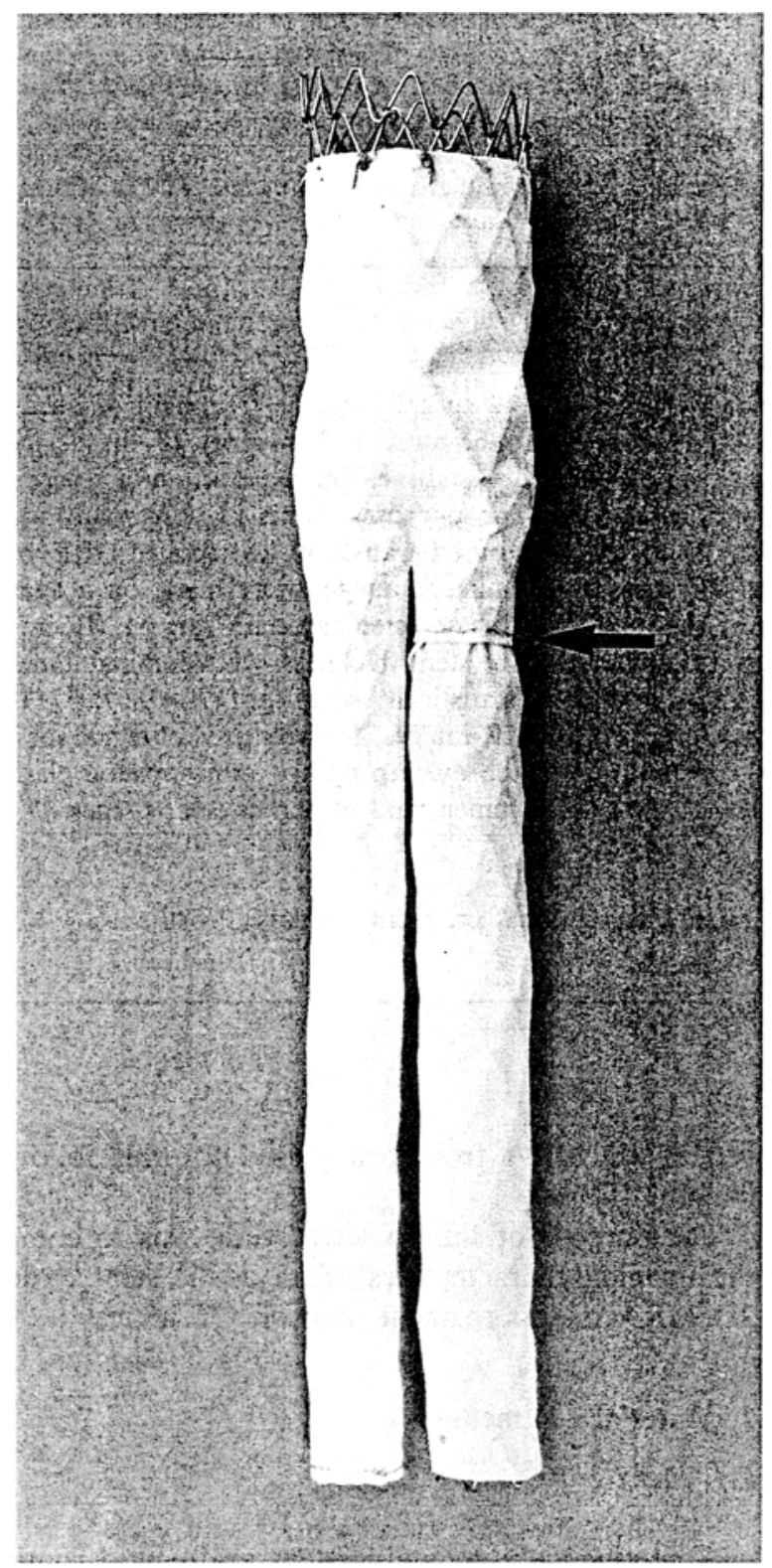

(a)

\subsection{In vitro Stent imaging}

The inflated stent graft was embedded into agar-gel, spiked in a concentration of 1:20 with both Gd-DTPA (Magnevist; Schering, Berlin, Germany) to simulate the intraluminal aortic signal during 3D MRA, as well as iodinated contrast (Visipaque, $270 \mathrm{mg} 1 \mathrm{ml}^{-1}$, Nycomed Imaging AS, Norway) to simulate the appearance of contrast-enhanced aortic blood.

All MR imaging was performed on a $1.5 \mathrm{~T} M R$ scanner (Signa EchoSpeed; GEMS, Milwaukee, Wis)

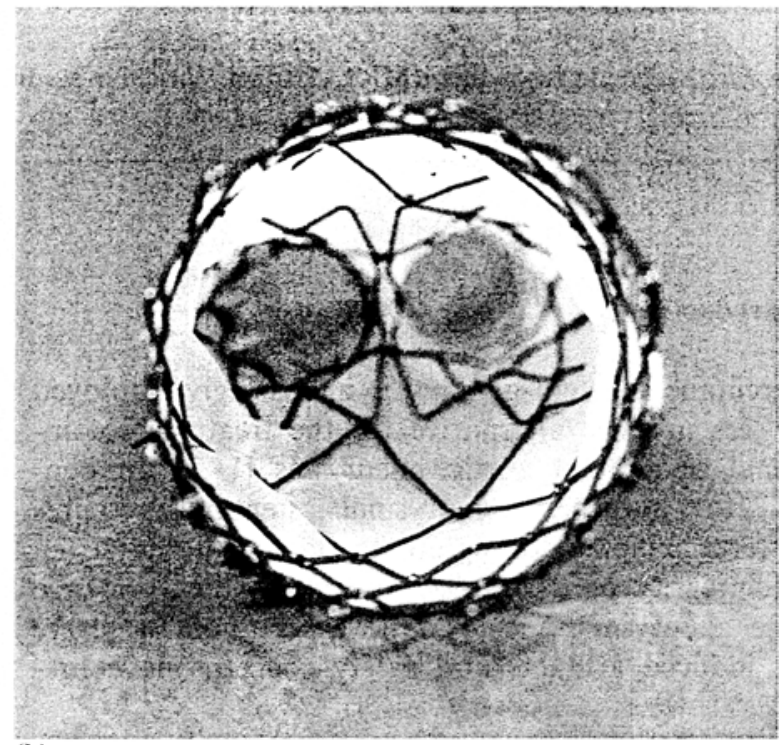

(b)

Fig. 1. Stent graft used for the endoluminal treatment of infrarenal aortic aneurysms (Vanguard; Boston Scientific, Oakland, NJ). (a) Frontal view. The graft has two components that are inserted separately and subsequently joined: the primary component consists of an aortic and iliac stent-graft with an attachment site for the secondary component. which is placed in the contralateral iliac artery. Junction of the two stent components (arrow). (b) View in the stent from the aortic part. 
using an anteroposterior phased-array surface coil for signal reception. Based on axial multiphase gradient-recalled-echo (ORE) localising images, a 3D Fourier transform fast spoiled GRE acquisitions was acquired consisting of 44 contiguous $2.5 \mathrm{~mm}$ coronal sections. The sequence employs a sampling bandwidth of +62.5 $\mathrm{kHz}$, a TR/TE of $4.0 / 1.9 \mathrm{~ms}$ and a flip of $40^{\circ}$. Combined with a $24 \times 24 \mathrm{~cm}^{2}$ field-of-view (FOV), a $256 \times$ 192 matrix provided an in-plane resolution of $0.9 \times 1.2$ $\mathrm{mm}^{2}$. Wall thickness and luminal diameter measurements were based on axial reconstructions.

CT imaging was performed on a 'Somatom Plus 4' scanner (Siemens Medical Systems, Erlangen, Germany), using the following parameters: $3 \mathrm{~mm}$ collimation width, $6 \mathrm{~mm}$ table speed (1:2 pitchratio), $240 \mathrm{~mA}$ and $120 \mathrm{kV}$. Helical CT scanning covered the whole stent-graft. Wall thickness and luminal diameter measurements were based on transaxial images, which were reconstructed every $3 \mathrm{~mm}$ (increment 3 ).

\subsection{In vitro Stent image analysis}

The stent-graft was rendered dark on MR images, and bright on the CT images. Stent wall thickness and diameters were measured on axial images at five identical locations:

1. proximal aortic portion traversing the platinum marker;

2. center of the aortic portion;

3. proximal iliac portion traversing the platinum marker; .

4. center portion of the iliac legs (left and right separately);

5. distal iliac portion traversing the platinum marker.

On each of the five selected images, wall thickness was measured at four points, each spaced $90^{\circ}$ from one another. At each location, two orthogonal luminal diameters, each connecting two opposite points were also measured. Measurements were based on signal intensity plots drawn orthogonal to the tangent of each measurement point. The luminal stent diameter was defined as the distance between the two inner points of half maximum/minimum signal intensity; wall thickness was defined as the distance between the inner and the outer point of half maximum/minimum signal intensity.

Statistical analysis of the CT and MR data was based on the paired Student's $t$-test, using a cut-off of 0.05 to signify statistical significance.

\section{Results}

The stent graft was well delineated on both MR and $\mathrm{CT}$ images. Reflecting its increased density, the stent was depicted as a bright ring on CT images. On MR images the stent structure created a signal void and was thus displayed as a dark ring. The low level of artifact on the 3D GRE images allowed the boundaries of the stent to be clearly seen (Fig. 2). The woven wires of the stent-graft were visible against the contrast enhanced agar-gel. Platinum markers exhibited artifacts resembling a starburst or ring pattern on $\mathrm{CT}$ images, whilst merely inducing a focally enlarged signal void on the MR images (Fig. 3). The $0.1 \mathrm{~mm}$ woven-polyester fabric could not been delineated on either MR or CT images.

Wall thickness measurements at the five locations of the stent graft overestimated the true stent thickness on both the MR and CT images. Between the two image sets, measurements revealed no statistically significant differences $(P=0.67)$ (Table 1). Merely wall thickness measurements obtained at the junction of the two stent components in the proximal portion of one iliac leg revealed a statistically significant difference: the platinum-induced artifacts simulated a wider appearance on MR images compared to CT images $(P=0.02)$. Reflecting the overestimation of wall thickness, luminal diameters were slightly underestimated on both MR and CT images. Measurement differences between MR and $\mathrm{CT}$ were not statistically significant $(P=0.85)$.

\section{Discussion and conclusion}

Currently spiral CTA is being propagated as the modality of choice for stent-graft follow-up [4]. Spiral CTA is fast, permitting coverage of a long vascular segment during the intra-arterial phase of a single intravenous contrast injection. The technique is non-invasive, has been shown to be more sensitive in the detection of perigraft flow than DSA, and permits reproducible stent-related measurements [4]. These measurements do appear to be fraught with some errors however, as documented by this study. Stent wall thickness was overestimated and luminal stent diameters were underestimated. In addition, the presence of metal can corrupt image quality in the form of starburst artifacts as shown by the stent platinum markers.

The results of this 'in vitro' experiment show that these stents can also be imaged with MRA. The very short echo time of less than $2 \mathrm{~ms}$, inherent to the fast 3D GRE acquisition, limits stent-related susceptibility artifacts, which are associated with other sequences $[8-10]$. The nitinol frame filaments of the stent-graft are identifiable on the individual sections and reformations as distinct areas of signal void, allowing for a detailed assessment of stent structure.

Similar to CTA, 3D MRA images tend to slightly overestimate the width of the stent graft wall. Based on half maximum/minimum measurements, the degree of wall thickness overestimation and associated underestimation of luminal stent diameters was no more pro- 


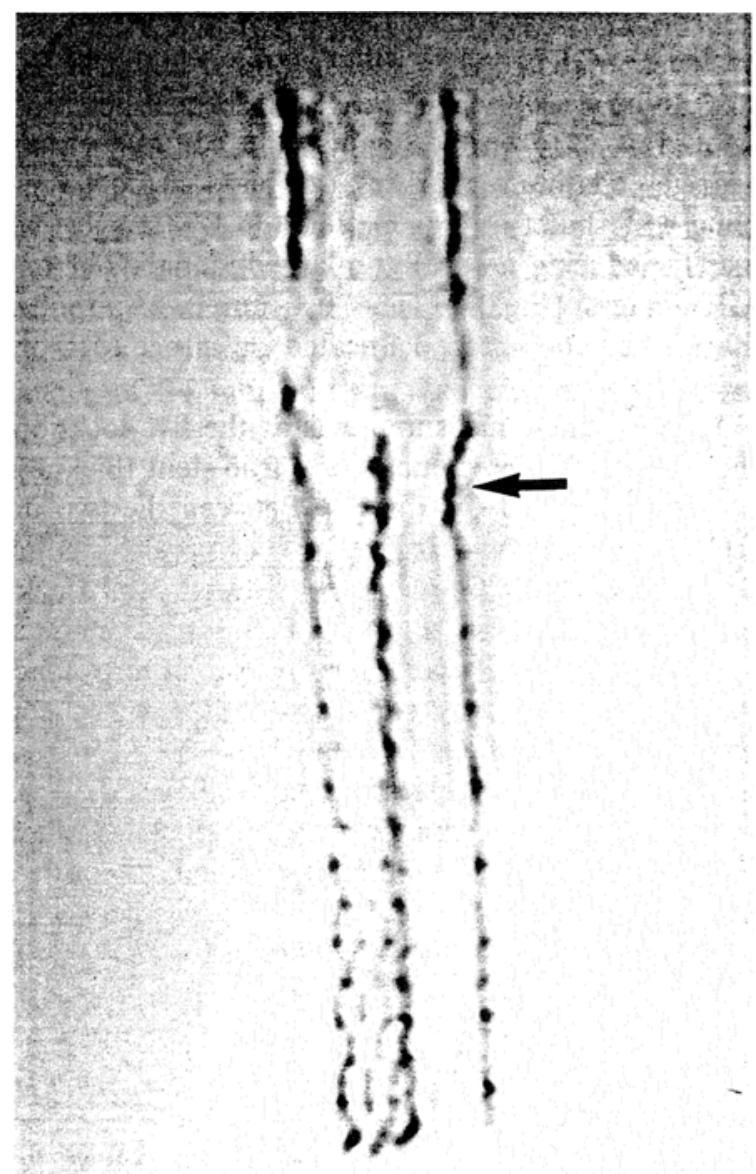

(a)

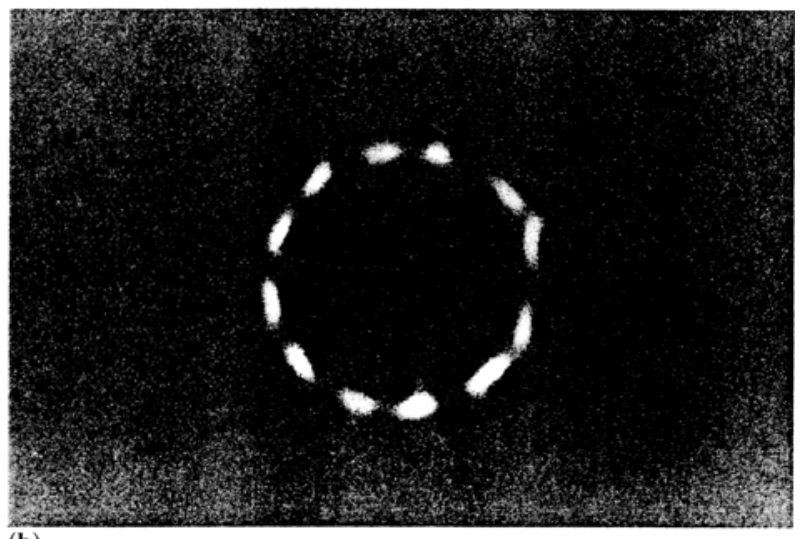

(b)

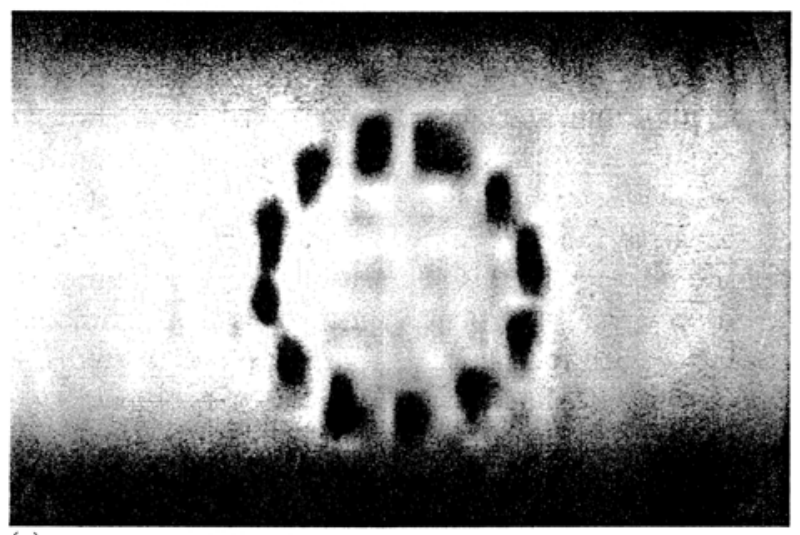

(c)

Fig. 2. Coronal 3D MRA source image (a) of the stent graft. The woven wires of the stent-graft are well visualized on the image against the contrast enhanced agar-gel. Platinum markers at the junction of the two stent components (arrow). Axial CT (b) and MR (c) images through the center of the aortic portion. Reflecting its increased density, the stent is depicted as a bright ring on axial CT images (b). On axial MR images (c) the stent structure created a signal void and was thus displayed as a dark ring.

nounced for MRA than CTA. In fact artifacts emanating from the platinum markers were considerably less severe on the MR-images. A wider area of signal loss was seen only at the insertion of the iliac stent leg into the aortic stent portion. This most likely reflects the overlap of two radio-opaque platinum markers. This artifact should under no circumstance be confused with a stenosis.

The introduction of metallic conducting materials into the scanner can potentially lead to hazardous ferromagnetic and thermal effects [11-13]. The Vanguard bifurcated stent graft can be imaged without risk 
of heating or magnetically induced device migration. Ferromagnetism could be excluded, as well as temperature increase during 3D GRE imaging [14].

After dispelling artifact- and safety-related concerns, potential advantages of 3D MRA over CTA regarding their application to post-stent follow-up imaging may be considered. Beyond the absence of ionizing radiation, paramagnetic contrast agents are not nephrotoxic $[15,16]$, permitting their repetitive use even in patients with impaired renal function. As many of the patients with aortic aneurysms have concomitant renal insufficiency [17-19] this aspect is of particular significance. Other advantages of 3D MRA over CTA, regarding assessment of the aorta with or without the presence of an endoluminal stent relate to the ability to image in the coronal plane. Better in plane resolution on coronal source images results in improved delineation of the aorta along its mostly coronal course [5]. Furthermore, the coronal acquisition plane extends the field-of-view to $40 \mathrm{~cm}$ thereby easily encompassing the pelvic arteries-an important aspect regarding both pre-procedural planning of stent delivery and post-stenting follow-up.

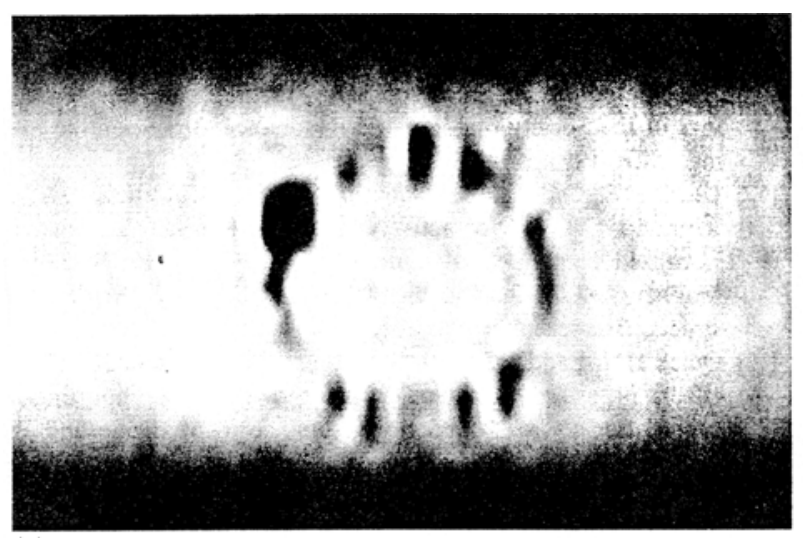

(a)

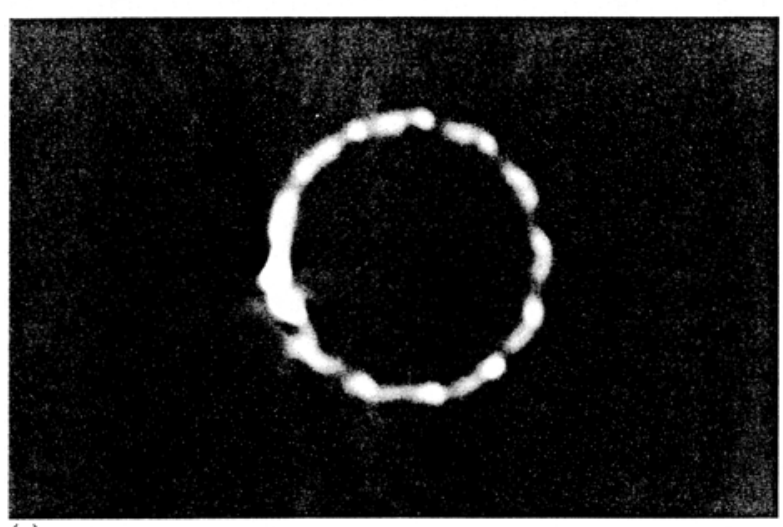

(c)
The outlined advantages do indeed suggest the use of 3D MRA for stent-graft follow-up imaging. A number of possible limitations must, however, be considered. Thus MRA cannot be performed on patients with contraindications to MR imaging, such as implanted pacemakers. Furthermore, the signal void around the stent-graft may hamper the detection of perigraft channels in the same way as in CT. This can be compensated for by closely following the size of the aneurysmal sac [4]. Any enlargement following stenting would indicate failure of the procedure. Finally it is crucial to point out that the favorable results documented with the Vanguard bifurcated stent graft cannot be generalized to other devices. Both artifact and safety characteristics are dependent on the composition of the individual stent graft. In view of considerable similarities between the different models, similarly favorable results can, however, be expected. These 'in vitro' experiments demonstrate that artifacts associated with the wall of the stent graft are not more pronounced on $3 \mathrm{D}$ MRA images than on CTA images. Merely those focal areas containing a platinum marker caused more signal drop-out on MRA data sets. 3D MRA images should

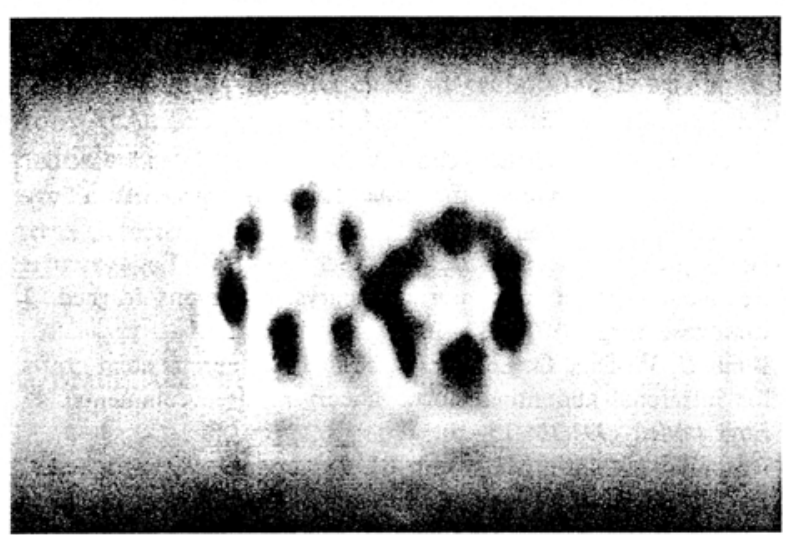

(b)

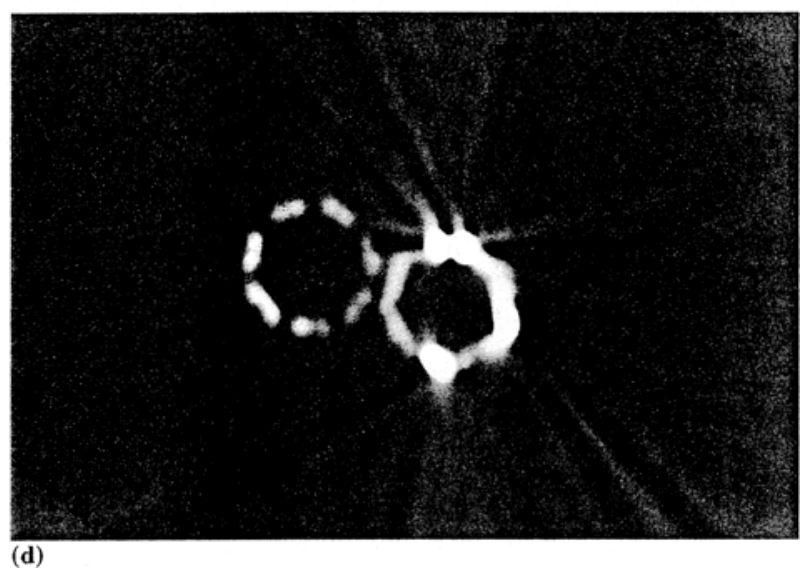

Fig. 3. MR (a, b) and CT (c, d) images of the stent-graft obtained at identical location traversing the platinum marker at the proximal aortic portion $(a, c)$ and traversing the platinum marker at the junction of the two stent components in the proximal iliac portion (b, d). The platinum markers induce a starburst pattern on CT images, whilst the platinum-induced signal voids simulated a widening of the stent wall on MR images. 
Table 1

Measurement of the wall thickness and diameters"

\begin{tabular}{llll}
\hline & $\mathrm{CT} / \mathrm{mm}$ & $\mathrm{MRI} / \mathrm{mm}$ & Stent dimensions/mm \\
\hline Diameter of prox. Aortic part & $21.9 \pm 1.1$ & $21.8 \pm 0.7(P=0.85)$ & 24 \\
Diameter of center of aortic part & $21.7 \pm 1.2$ & $21.7 \pm 0.6(P=0.93)$ & 24 \\
Diameter prox. iliac portion & & & \\
Attached iliac section & $7.4 \pm 0.2$ & $7.6 \pm 1.1(P=0.83)$ & 10 \\
Inserted iliac section & $6.9 \pm 0.5$ & $5.5 \pm 0.8(P=0.02)$ & 10 (junction) \\
Diameter center of iliac legs & & & 10 \\
Attached iliac section & $7.3 \pm 0.2$ & $7.7 \pm 1.0(P=0.77)$ & 12 \\
Inserted iliac section & $9.2 \pm 0.6$ & $9.2 \pm 1.0(P=0.96)$ & 10 \\
Diameter distal iliac legs & & & 12 \\
Attached iliac section & $7.3 \pm 0.3$ & $7.6 \pm 0.9(P=0.81)$ & 0.35 \\
Inserted iliac section & $8.8 \pm 0.6$ & $8.5 \pm 0.6(P=0.80)$ & 0.95 \\
Wall thickness & $2.3 \pm 0.4$ & $2.4 \pm 0.8(P=0.67)$ & $3.4 \pm 1.3(P=0.02)$ \\
Platinum markers & $2.0 \pm 0.5$ & & \\
\hline
\end{tabular}

\footnotetext{
a The stent-graft had the following sizes: diameter of the aortic part: $24 \mathrm{~mm}$; diameter of the attached iliac section: $10 \mathrm{~mm}$; diameter of the
} inserted iliac section: $10 \mathrm{~mm}$ (junction), $12 \mathrm{~mm}$ (free end)

thus permit a comprehensive assessment of the arterial lumen as well as of perivascular tissues, thereby evolving into an alternative for the follow-up of stent-grafting procedures.

\section{References}

[1] Parodi JC. Marin ML. Veith FJ. Transfemoral, endovascular stented graft repair of an abdominal aortic aneurysm. Arch Surg 1995:130:549-52.

[2] Parodi JC, Barone A, Piraino R, Schonhoiz C. Endovascular treatment of abdominal aortic aneurysms: lessons learned. J Endovasc Surg 1997;4:102-10.

[3] Blum U, Voshage G, Lammer J, et al. Endoluminal stent-grafts for infrarenal abdominal aortic aneurysms [see comments]. N Engl J Med 1997;336:13-20.

[4] Rozenblit A, Marin ML, Veith FJ, Cynamon J, Wahi SI. Bakal $\mathrm{CW}$. Endovascular repair of abdominal aortic aneurysm: value of postoperative follow-up with helical CT. Am J Roentgenol 1995; 165:1473-9.

[5] Thurnher SA, Dorffner R, Thurnher MM, et al. Evaluation of abdominal aortic aneurysm for stent-graft placement: comparison of gadolinium-enhanced MR angiography versus helical CT angiography and digital subtraction angiography. Radiology 1997;205:341-52.

[6] Cragg AH, De Jong SC, Barnhart WH, Landas SK, Smith TP. Nitinol intravascular stent: results of preclinical evaluation. Radiology 1993;189:775-8.

[7] Blum U, Langer M, Spillner G, et al. Abdominal aortic aneurysms: preliminary technical and clinical results with transfemoral placement of endovascular self-expanding stent-grafts [see comments]. Radiology 1996:198:25-31.

[8] Teitelbaum GP, Bradley WG Jr, Klein BD. MR imaging artifacts, ferromagnetism, and magnetic torque of intravascular filters, stems, and coils. Radiology 1988;166:657-64.

[9] Teitelbaum GP. Raney M, Carviin MJ, Matsumoto AH, Barth $\mathrm{KH}$. Evaluation of ferromagnetism and magnetic resonance imaging artifacts of the Strecker tantalum vascular stent. Cardiovasc Iotervent Radiol 1989:12:125-7.

[10] New PFI, Rosen BR, Brady TJ, et al. Potential hazards and artifacts of ferromagnetic and nonferromagnetic surgical and dental materials and devices in nuclear magnetic resonance imaging. Radiology 1983;147:139-48.

[1-1] Maier SE, Wildermuth SE, Darrow RD, Watkins RD, Debatin JF, Dumoulin CL. Safety of MR Tracking catheters. Proceedings of the Society of Magnetic Resonance, 3rd Scientific Meeting, Nice, 1995. p. 497.

[12] Ladd ME, Quick HH, Debatin JF, von Schulthess GK, Mc Kinnon GC. Safety of actively visualized vascular guidewiresLocalized heating. 14th annual meeting European Society for Magnetic Resonance in Medicine and Biology. Book of abstracts, Brussels. 1997, p. 281.

[13] Quick HH, Ladd ME, von Schulthess GK, Debatin JF, Heating effects of an intravascular catheter. 14th annual meeting European Society for Magnetic Resonance in Medicine and Biology. Book of abstracts. Brussels, 1997, p. 563.

[14] Hilfiker PR, Pfammatter T, Hany TF, Lachat M, Debatin JF, 3D MRA in patients with abdominal aortic aneurysm post stent-grafting. 6th scientific meeting ISMRM. Book of abstracts, Sydney, 1998, p. 175.

[15] Niendorf HP, Hauenstein J, Cornelius I, Alhassan A, Clauss W. Safety of gadolinium-DTPA: extended clinical experience. Magn Reson Med 1991;22:222-8.

[16] Nelson KL, Gifford LM, Lauber-Huer C, Gross CA, lesser TA Clinical safety of gadopentetate dimeglumine. Radiology 1995; 196:439-43.

[17] Koskas F, Kieffer E. Long-term survival after elective repair of infrarenal abdominal aortic aneurysm: results of a prospective multicentric study. Association for Academic Research in Vascular Surgery (AURC). Ann Vase Surg 1997;11:473-81.

[18] Muluk SC, Painter L, Sile S, et al. Utility of clinical pathway and prospective case management to achieve cost and hospital stay reduction for aortic aneurysm surgery at a tertiary care hospital. J Vasc Surg 1997;25:84-93.

[19] Komori K, Kuma S, Eguchi D, et al. Surgical strategy of abdominal aortic aneurysm with preoperative renal failure. Eur $\mathrm{J}$ Vasc Endovasc Surg 1997:14:105-8. 